\title{
Potenciais evocados auditivos de longa latência e processamento auditivo central em crianças comalterações de leitura e escrita: Dados preliminares
}

\section{Long latency auditory evoked potentials and central auditory processing in children with reading and writing alterations: Preliminary data}

\author{
Aparecido José Couto Soares', Seisse Gabriela Gandolfi Sanches², Ivone Ferreira Neves-Lobo 3 , Renata Mota Mamede Carvallo4, \\ Carla Gentile Matas 4 , Maria Sílvia Cárnio5.
}

1) Mestrando em Ciências da Reabilitação pela FMUSP. Fonoaudiólogo do Departamento de Fisioterapia, Fonoaudiologia e Terapia Ocupacional da FMUSP. 2) Doutora em Ciências da Reabilitação pela FMUSP. Fonoaudióloga do Departamento de Fisioterapia, Fonoaudiologia e Terapia Ocupacional da FMUSP.

3) Doutora em Ciências na Área de Fisiopatologia Experimental pela FMUSP. Fonoaudióloga do Departamento de Fisioterapia, Fonoaudiologia e Terapia Ocupacional da FMUSP.

4) Livre Docente. Professora Associada FMUSP.

5) Doutora. Professora Assistente FMUSP.

Instituição: Departamento de Fisioterapia, Fonoaudiologia e Terapia Ocupacional da Faculdade de Medicina da Universidade de São Paulo (FMUSP). São Paulo/SP - Brasil

Endereço para correspondência: Aparecido José Couto Soares - Rua Cipotânea, 51 - Cidade Universitária - São Paulo / SP - Brasil - CEP: $05360-160$ - Telefone: (+55 11) 3091-8412 - Fax: (+55 11) 3091-7714 - E-mail: ajcsoares@usp.br

Artigo recebido em 29 de Junho de 2011. Artigo aprovado em 14 de Agosto de 2011

\section{RESUMO}

Introdução: Atualmente, admite-se que indivíduos com alterações de leitura e escrita podem apresentar atraso no desenvolvimento das habilidades auditivas, o que pode interferir no processo de aprendizagem. A avaliação das habilidades auditivas pode ocorrer de forma comportamental, por meio dos testes do processamento auditivo central (PAC), ou por meio da avaliação eletrofisiológica, destacando-se os potenciais evocados auditivos de longa latência (PEALL). A utilização dos PEALL como meio de avaliação complementar de indivíduos com alterações de leitura e escrita pode se tornar um importante dado tanto para uma caracterização mais detalhada das alterações, como para o direcionamento terapêutico dessa população.

Objetivo: Caracterizar o PAC e o PEALL em crianças com alterações de leitura e escrita.

Método: Pesquisa aprovada pela Comissão de Ética da Instituição sob no 305/10. A avaliação do PAC e do PEALL foi realizada em 12 crianças com idade entre oito e 12 anos (média de 10,6 anos), com alteração de leitura e escrita confirmada em avaliação específica.

Resultados: As habilidades do PAC mais alteradas foram ordenação temporal e figura-fundo para sons linguísticos. Foram encontrados resultados alterados no P300 e no MMN.

Conclusão: Os indivíduos com alterações de leitura e escrita apresentaram desempenho abaixo do esperado em testes do PAC. O MMN possibilitou uma melhor caracterização da função auditiva desta população. Houve indícios de associação entre resultados do PAC e alteração nos PEALL.

Palavras-chave: aprendizagem, audição, avaliação.

\section{SUMMARY}

Introduction: Presently, it is admitted that individuals with reading and writing alterations may present delay in the development of listening skills, which may interfere in the learning process. The assessment of the listening skills can occur in a behavioral way, through central auditory processing (CAP) tests, or by electrophysiological assessment highlighting the long latency auditory evoked potentials (LLAEP). The use of the LLAEP as a means of complementary assessment of individuals with reading and writing alterations can become an important data both for further characterization of the alterations, as for the therapeutic guidance of this population. Objective: Characterize the CAP and the LLAEP in children with reading and writing alterations.

Method: Research approved by the Institution's Ethic Commission under $\mathrm{n}^{-}$305/10. The assessment of CAP and LLAEP was performed in 12 children aged between 8 and 12 years old (average of 10,6 years), with reading and writing alterations confirmed in specific evaluation.

Results: The most altered CAP skills were temporal ordination and figure-ground for linguistic sounds. There were found altered results in P300 and in MMN.

Conclusion: The individuals with reading and writing alterations performed below the expected on CAP tests. The MMN allowed a better characterization of the auditory function of this population. There was evidence of association between the CAP results and the alteration of the LLAEP.

Keywords: learning, hearing, assessment. 


\section{INTRODUÇÃO}

As alterações de leitura e escrita caracterizam-se pela dificuldade na aquisição e/ou desenvolvimento da linguagem escrita, envolvendo déficit tanto de decodificação fonológica como de compreensão da linguagem oral e/ou escrita (1).

Atualmente, admite-se que indivíduos com alterações de leitura e escrita podem apresentar atraso no desenvolvimento das habilidades auditivas, impedindo o adequado processamento da informação e interferindo no processo de aprendizagem (2). Diversos estudos (3, 4, 5) correlacionam alterações em habilidades de leitura e escrita com possíveis transtornos do processamento auditivo (central) (PAC).

Pesquisas $(6,7)$ apontam que, entre as comorbidades que comumente apresentam alterações de PAC, os transtornos de aprendizagem são as mais frequentes. As dificuldades estariam em processar características temporais de estímulos de diferentes modalidades sensoriais, quando apresentados de maneira rápida e em sequência (8).

Cabe ressaltar que a avaliação das habilidades auditivas pode ocorrer de forma comportamental, por meio dos testes do PAC, ou por meio da avaliação eletrofisiológica, que reflete principalmente a atividade da via auditiva frente ao estímulo desde a região do tronco encefálico até a do córtex, possibilitando também a avaliação de habilidades de processamento sequencial de informações, memória imediata e/ou tomada de decisões $(9,10)$. No espectro da avaliação eletrofisiológica da audição destaca-se a investigação dos potenciais evocados auditivos de longa latência (PEALL).

Os PEALL refletem a atividade neuroelétrica da via auditiva nas regiões do tálamo e córtex auditivo, estruturas que envolvem as funções de discriminação, integração e atenção, fornecendo informações sobre o funcionamento do Sistema Nervoso Auditivo Central (SNAC).

O P300 e o Mismatch Negativity (MMN) são potenciais evocados auditivos de longa latência (PEALL), pois surgem entre 80 e 750 ms (11) após a apresentação do estímulo e captam respostas geradas em regiões corticais. São eliciados em tarefas oddball (estímulo alvo), na discriminação de dois estímulos diferentes, sendo um apresentado de maneira frequente e outro introduzido de maneira aleatória, denominado estímulo raro. Estes potenciais são utilizados na investigação de habilidades cognitivas como discriminação e atenção, por este motivo podem ser denominados de potenciais cognitivos.
A utilização dos PEALL como meio de avaliação complementar de indivíduos com alterações de leitura e escrita pode se tornar um importante dado tanto para uma caracterização mais detalhada das alterações, como para o direcionamento terapêutico dessa população.

Entretanto, ainda são escassos na literatura nacional, estudos que investiguemalém dos aspectos comportamentais doPAC, os PEALL e suas relações com as alterações de leitura e escrita. Baseado no exposto, o objetivo deste trabalho foi caracterizar o PAC e os PEALL em crianças com alterações de leitura e escrita.

\section{MÉTODO}

Esta pesquisa foi aprovada pela Comissão de Ética para Análise de Projetos de Pesquisa do Hospital das Clínicas da FMUSP (CAPPesq- Protocolo de Pesquisa no 305/10).

Os testes auditivos (comportamentais e eletrofisiológicos) foram realizados em 12 crianças com idade entre 8 e 12 anos (média de 10,6 anos), escolaridade entre $3^{\underline{a}}$ e $6^{\mathrm{a}}$ séries do Ensino Fundamental, com alteração de leitura e escrita confirmada em avaliação específica realizada no Laboratório de Investigação Fonoaudiológica (LIF) em Leitura e Escrita da FMUSP. Nesta avaliação foram investigados os seguintes aspectos: linguagem oral, consciência fonológica, memória operacional fonológica, nomeação automática rápida, leitura e escrita.

Os critérios de inclusão adotados para composição da amostra foram: presença de alterações de leitura e escrita confirmadas por avaliação fonoaudiológica específica, limiares auditivos normais ( $\leq 20 \mathrm{~dB}$ NA nas frequências de 0.25 a $8 \mathrm{kHz}$ ) e curva timpanométrica Tipo A no momento da avaliação.

\section{Procedimentos}

Após assinatura do Termo de Consentimento Livree Esclarecido pelos pais e/ou responsáveis, as crianças foram submetidas inicialmente a imitanciometria, utilizando o analisador de orelha média modelo GSI 33, da marca Grason Stadler (Versão 2-ANSI 1987). Em seguida, foram realizadas a audiometria tonal por via aérea nas frequências de 0,25 a $8 \mathrm{kHz}$ e audiometria vocal incluindo Limiar de Reconhecimento de Fala (LRF) e Índice de Reconhecimento de Fala (IRF), utilizando para tanto o audiômetro modelo GSI 61, da marca Grason Stadler (ANSI S3,6-1989; ANSI S3,43-1992; IEC 645-1,1992; IEC 645 - 2, 1993; ISO 389; UL 544).

Após o preenchimento dos critérios de inclusão, foi realizada a avaliação do processamento auditivo (central) 
em cabina audiométrica com a gravação dos estímulos em compact disc (12), reproduzido por um CDPlayeracoplado ao audiômetro, apresentados por meio de fones de ouvido. Para tanto, foi utilizada uma bateria de testes comportamentais padronizados, contendo testes temporais (que avaliam habilidades auditivas de ordenação temporal e resolução temporal), teste monótico (que avalia habilidade auditiva de fechamento auditivo) e testes dicóticos (que avaliam habilidade auditiva de figura-fundo). Primeiramente, foram aplicados os testes padrão de frequência (TPF) e padrão de duração (TPD), de forma binaural, ou seja, com estímulos apresentados nas duas orelhas ao mesmo tempo, na intensidade de 50 dB NS sobre o LRF. O próximo teste aplicado foi o Teste Dicótico de Dissílabos Alternados (SSW), e o Teste Dicótico de Dígitos (DD), a mbos aplicados a 50 dB NS. Foi utilizado, também, o Teste monótico de Fala com Ruído Branco (TFRB) na relação sinal/ruído +20 dB (conforme padronizado para o equipamento utilizado), apresentado de forma monoaural em intensidade de $40 \mathrm{~dB}$ NS. Para finalizar, foi realizado o Teste Gaps in Noise (GIN), em intensidade de $50 \mathrm{~dB} \mathrm{NS}$, com os estímulos apresentados de forma binaural.

Em outra sessão foram realizados os procedimentos eletrofisiológicos, utilizando-se os potenciais evocados auditivos de longa latência (P300 e Mismatch Negativity-MMN). Para tanto foi utilizado o equipamento Sistema Portátil Modelo Opti-Amp 8002 da marca Intelligent Hearing, com fones TDH- 49 , estando a criança sentada confortavelmente em uma poltrona reclinável.

Para a realização do $\mathrm{P300}$ e do $\mathrm{MMN}$, a disposição dos eletrodos seguiu a norma internacional 10/20, sendo o eletrodo ativo posicionado em $\mathrm{Cz}$ (vértex), os eletrodos referência em A1 e A2 (mastoides esquerda e direita, respectivamente), e o eletrodo terra ( $\mathrm{Fpz}$ ) na fronte. Foram utilizados estímulos acústicos tone burst em $1000 \mathrm{~Hz}$ (estímulo frequente) e $1500 \mathrm{~Hz}$ (estímulo raro) a $80 \mathrm{~dB} \mathrm{NA}$, apresentados aleatoriamente por meio de fones TDH- 49 num paradigma oddball, em velocidade de apresentação de 1,1 estímulos por segundo, com uma probabilidade de ocorrência do estímulo raro de $20 \%$. Os testes foram realizados separadamente por orelhas.

Durante a realização do $\mathrm{P} 300$, as crianças foram instruídas a prestar atenção nos estímulos raros, contando-os em voz alta. Para realização do MMN, foi utilizado um distrator visual (filme infantil) a fim de evitar que a criança tivesse a atenção voltada para o estímulo raro.

Para a avaliação do processamento auditivo (central) e a avaliação eletrofisiológica foram adotados os padrões de normalidade estabelecidos na literatura especializada, conforme a faixa etária $(12,13,14)$. O indivíduo foi considerado alterado quando, pelo menos, uma orelha apresentou alte- ração, tanto para a avaliação comportamental quanto para a eletrofisiológica.

Os resultados obtidos foram submetidos à análise estatística. Para a comparação entre as latências foi utilizado o teste não paramétrico de Wilcoxon e, para verificar a associação entre as variáveis, foi utilizado o teste do Quiquadrado. Foi adotado o nível de significância de 5\%.

\section{RESULTADOS}

Observou-se que dos 12 indivíduos, 11 (92\%) apresentaram alterações em algumas habilidades do processamento auditivo, sendo que as mais alteradas foram às habilidades de ordenação temporal e figura-fundo (Gráfico 1).

Para a análise estatística das latências do P300 e do MMN foi utilizado o teste não paramétrico de Wilcoxon, pois a distribuição dos dados não respeita a normalidade (Kolmogorov-Smirnov < 0,05). Não houve diferença significante na comparação entre as orelhas direita e esquerda para as latências em nenhum dos potenciais evocados auditivos avaliados (Tabela 1).

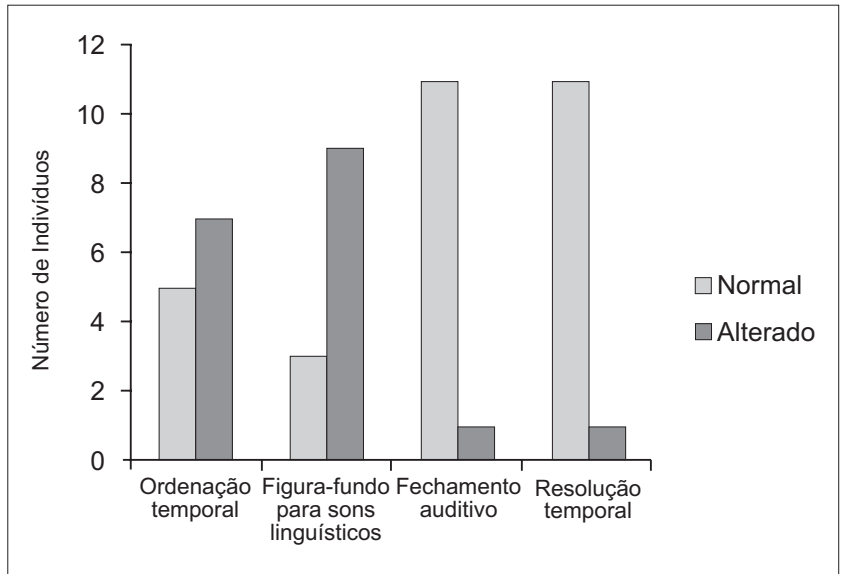

Gráfico 1. Número de indivíduos alterados por habilidades do PAC.

Tabela I. Análise descritiva e resultados do teste nãoparamétrico de Wilcoxon para a comparação das latências entre as orelhas.

\begin{tabular}{lcccc}
\hline & \multicolumn{2}{c}{ P300 } & \multicolumn{2}{c}{ MMN } \\
& Orelha & Orelha & Orelha & Orelha \\
& Direita & Esquerda & Direita & Esquerda \\
\hline Média & 350,33 & 330,08 & 304,92 & 324,67 \\
Mediana & 350,50 & 330,00 & 289,50 & 322,50 \\
Mínimo & 295 & 257 & 253 & 246 \\
Máximo & 423 & 426 & 395 & 375 \\
Desvio-padrão & 41,64 & 47,14 & 44,87 & 44,35 \\
p-valor & \multicolumn{2}{c}{0,151} & \multicolumn{2}{c}{0,227} \\
\hline
\end{tabular}




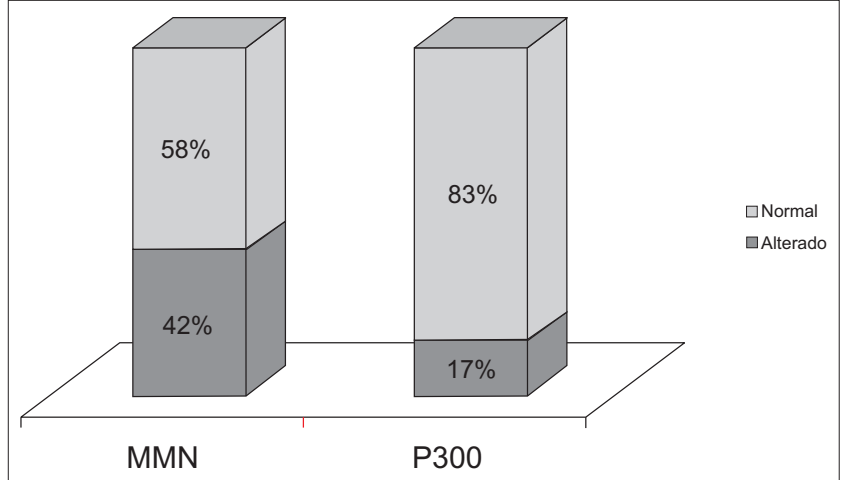

Gráfico 2. Desempenho dos indivíduos na avaliação dos PEALL.

A distribuição dos resultados obtidos com os PEALL revela que as crianças apresentaram mais alterações no MMN (42\%) do que no P300 (17\%) (Gráfico 2). O teste Qui-quadrado revelou não haver associação entre o desempenho no MMN e no P300 ( $\mathrm{X}^{2}=0,069, \mathrm{gl}=1$, $\mathrm{p}$-valor $=0,793)$.

Na comparação entre os resultados das avaliações eletrofisiológicas e comportamental observou-se que 71\% das crianças que apresentaram alteração na habilidade auditiva de ordenação temporal também apresentaram alteração em algum dos PEALL. Além disso, 44\% dos indivíduos com alterações de figura-fundo também apresentaram alteração nos PEALL.

Todavia, o teste do Qui-quadrado revelou não haver associação entre o desempenho nos PEALL e nas habilidades do PAC, como pode ser observado na Tabela 2.

\section{DISCUSSÃO}

A avaliação das habilidades auditivas tem se tornado um importante instrumento a ser considerado na gama de procedimentos utilizados na avaliação de indivíduos com alterações de leitura e escrita. Assim sendo, este estudo objetivou caracterizar o PAC e os PEALL em crianças com alterações de leitura e escrita.

Os dados obtidos no presente estudo indicam que é comum encontrar resultados alterados na avaliação do PAC em indivíduos com alterações de leitura e escrita, conforme descrito também em outros estudos $(15,16,17)$. As habilidades auditivas de figura-fundo para sons linguísticos e de ordenação temporal foram as mais alteradas, corroborando a literatura especializada $(2,3,7,18)$. Os déficits em habilidades temporais reforçam a hipótese de que tal prejuízo poderia estar relacionado a uma alteração no processamento de "elementos acústicos
Tabela 2. Resultados doteste Qui-quadrado paraa comparação entre o desempenho nos PEALL e nas habilidades do PAC.

\begin{tabular}{|c|c|c|c|}
\hline & $X^{2}$ & gl & p-valor \\
\hline MMN*PAC & 0,779 & I & 0,377 \\
\hline P300 * PAC & 0,218 & | & 0,64 \\
\hline MMN* Ordenaçãotemporal & 1,656 & । & 0,198 \\
\hline MMN*Figura-fundo para sons linguísticos & 1,029 & | & 0,31 \\
\hline MMN*Fechamentoauditivo & |,527 & । & 0,217 \\
\hline MMN*Resoluçãotemporal & 1,527 & | & 0,217 \\
\hline P300 * Ordenaçãotemporal & $|, 7| 4$ & | & 0,19 \\
\hline P300 * Figura-fundo para sons linguísticos & 0,800 & । & 0,37 \\
\hline P300 *Fechamento auditivo & 0,218 & | & 0,64 \\
\hline P300 * Resoluçãotemporal & 0,218 & | & 0,6 \\
\hline
\end{tabular}

curtos" como as consoantes, que são caracterizadas por rápida transição de formantes, o que levaria, consequentemente, a uma dificuldade em associar letras aos seus sons específicos (8).

Em estudo descrito na literatura nacional (19) observou-se que déficits no processamento temporal produzem dificuldades de discriminação, coordenação e integração no domínio verbal e não-verbal envolvendo nomeação, repetição, memória de trabalho e memória de longo prazo. Esses resultados também foram observados em outra pesquisa brasileira (20) que investigou as habilidades de consciência fonológica e de processamento auditivo em crianças com e sem alterações de leitura e escrita.

Entretanto, em outro trabalho (3), a correlação encontrada entre leitura e PAC foi considerada fraca. Uma das hipóteses sustentada para este achado é a de que o desempenho abaixo do esperado em testes de processamento temporal auditivo seja consequência de fatores não considerados no estudo em questão, como a atenção, as habilidades cognitivas e a memória de trabalho.

Por sua vez, os déficits em figura-fundo podem estar relacionados a alguns obstáculos enfrentados pelo escolar em sala de aula. A dificuldade na realização de tarefas na presença de ruído e sons competitivos pode implicar em dificuldades de assimilação do conteúdo ensinado, particularmente se houver alterações de memória associada. Dessa forma, torna-se difícil para o escolar, por exemplo, entender o que diz o professor, além do estresse promovido pela situação de ruído (21).

Além disso, tais dados reforçam a concepção de que o escolar com algum transtorno de aprendizagem apresenta alteração na capacidade e manutenção da atenção auditiva, o que prejudica a habilidade de figura-fundo para sons verbais (22). Sendo assim, destaca-se a necessidade de estudos que busquem correlacionar as habilidades de 
memória e atenção com os testes que avaliam o PAC, para a obtenção de resultados mais conclusivos.

Em relação aos PEALL, especificamente o P300, o número pequeno de alterações encontradas se deve, provavelmente, à grande variabilidade intersujeitos nos valores de normalidade deste teste. Resultado semelhante já foi descrito na literatura nacional (23). No que diz respeito ao $\mathrm{MMN}$, os dados sugerem que este teste para possibilitou uma melhor caracterização da função auditiva desta população. Entretanto, considera-se uma limitação deste estudo a amostra reduzida, fator que não permite generalizações.

Os PEALL trazem contribuições importantes na investigação de algumas habilidades cognitivas envolvidas no processamento da informação acústica (atenção, discriminação e memória), habilidades essenciais para o desenvolvimento adequado da leitura e escrita (24). Ressalta-se, porém, a necessidade de pesquisas adicionais, que possibilitariam inferir sobre qual potencial seria mais adequado para investigar determinadas habilidades de processamento auditivo (central), nesta população especifica.

Ao considerarmos que a leitura é um modo particular de aquisição de informações formada pelo conjunto de eventos que se passam no tempo, especula-se que a integridade dos mecanismos fisiológicos auditivos exerce um papel fundamental na percepção da fala, no aprendizado e na compreensão da linguagem e, consequentemente, é pré-requisito na aquisição da leitura e da escrita (25). Sendo assim, torna-se cada vez mais importante a avaliação das habilidades auditivas dessa população, pois tais alterações, quando detectadas precocemente, permitem a adequada orientação aos pais e professores, além de possibilitar o encaminhamento e intervenção especializada o mais breve possível.

\section{CONCLUSÃO}

Os indivíduos com alterações de leitura e escrita apresentaram desempenho abaixo do esperado em testes de processamento auditivo (central). Foram encontradas, também, alterações nos PEALL, sendo que o MMN possibilitou uma melhor caracterização da função auditiva desta população. Houve indícios de associação entre alteração na habilidade auditiva de ordenação temporal e alteração nos PEALL.

\section{REFERÊNCIAS BIBLIOGRÁFICAS}

1. Santos MTM, Navas ALGP. Distúrbios de leitura e escrita: teoria e prática. Barueri: Manole, 2002.
2. Pinheiro FH, Capellini SA. Treinamento auditivo em escolares com distúrbio de aprendizagem. Pró-Fono R. Atual. Cient. 2010, 22(1):49-54.

3. Engelmann, L \& Ferreira, MIDC. Avaliação do Processamento auditivo em crianças com dificuldades de aprendizagem. Rev Soc Bras Fonoaudiol. 2009, 14(1):6974.

4. Murphy CFB, Schochat E. Correlações entre leitura, consciência fonológica e processamento temporal auditivo. Pró-Fono R Atual Cient. 2009, 21(1):13-8.

5. Neves, I. F.; Schochat, E. Maturação do processamento auditivo em crianças com e sem dificuldades escolares. PróFono R. Atual. Cient, Barueri (SP). 2005, 17(3):311-320.

6. Borges CF, Schochat E. Fatores de risco para o Transtorno do Processamento Auditivo. Temas sobre Desenvol. 2005, 14:83-8.

7. Fortes AB, Pereira LD, Azevedo MF. Resolução temporal: análise em pré escolares nascidos a termo e pré-termo.PróFono R. Atual. Cient. 2007, 19(1):87-96.

8. Habib M. The neurological basis of developmental dyslexia. An overview and working hypothesis. Brain. 2000, 123:2373-99.

9. Musiek FE, Baran JA, Pinheiro ML. Behavioral and electrophysiological test procedures. In: Musiek FE, Baran JA, Pinheiro ML. Neuroaudiology: case studies. San Diego: Singular Publishing Group; 1994. cap. 2. p. 7-28.

10. Halgren E, Squires NK, Wilson CL, Rohrbaugh JW, Babb TL, Crandall PH. Endogenous potentials generated in the human hippocampal formation and amygdala by infrequent events. Science. 1980, 210(4471):803-5.

11. Ruth RA, Lambert PR. Auditory evoked potentials. Otolaryngol Clin North Am. 1991, 24(2):349-70.

12. Pereira LD, Schochat E. Testes auditivos comportamentais para avaliação do processamento auditivo central. Editora Pró Fono; 2011. 82p.

13. McPherson DL. Late potencials of the auditory system (evoked potencials). San Diego: Singular Press; 1996.

14. Brossi AB, Borba KC, Garcia CFD, Reis ACMB, Isaac ML. Verificação das respostas do mismatch negativity (MMN) em sujeitos adultos normais. Rev Bras Otorrinolaringol. 2007, 73(6):793-802.

15. Moore DR, Ferguson MA, Halliday LF, Rilley A.Frequency 
discrimination in children: Perception, learning and attention. Hearing Research. 2008, 238:147-54.

16. Silver CH, Ruff RM, Iveson GL, Barth JT, Broshek,DK, Bush SS, Koffer SP, Reynolds, CR. Learning disabilities:The need for neuropsycological evaluation. Archives of Clinical Neuropsychology. 2008, 23:217-19.

17. Dawes P, Bishop DVM, Sirimanna T, Bamiou DE. Profileand aetiology of children diagnosed with auditiry processingdisorder (APD). Int J Pediatr Otorhinolaryngol. 2008, 72:483-489.

18. Abdo AGR, Murphy CFB, Schochat E. Habilidades auditivas em crianças com dislexia e transtorno do déficit de atenção e hiperatividade. Pró-Fono R Atual Cient. 2010, 22(1):25-30.

19. Capovilla F \& Capovilla A. Intervenção em dificuldades de leitura e escrita com tratamento de consciência fonológica. In: Santos MTM, Navas ALGP. Distúrbios de Leitura e Escrita: teoria e prática. São Paulo: Manole, 2002. cap 7, p.225-261.

20. Garcia L, Campos P, Padovani R. Associação entre a avaliação de habilidades de consciência fonológica e de processamento auditivo em crianças com e sem distúrbios de aprendizagem. Rev Fono Atual, São Paulo. 2005, 8(31), 4-8.

21. Garcia VL, Pereira LD, Fikuda Y. Selective attention: psi performance in children with learning disabilities. Rev Bras Otorrinolaringol. [online]. 2007, 73(3):404-411. ISSN 00347299.

22. Pinheiro FH, Oliveira AD de, Cardoso ACV, Capellini SA. Testes de escuta dicótica em escolares com distúrbio de aprendizagem. Braz J Otorhinolaryngol. (Impr.) [online]. 2010, 76(2):257-262.

23. Farias LS, Toniolo IF, Coser PL. P300: avaliação eletrofisiológica da audição em crianças sem e com repetência escolar. Rev Bras Otorrinolaringol. [online]. 2004, 70(2):194-199. ISSN 0034-7299.

24. Vieira PAC. Influências das desordens de processamento auditivo na avaliação neuropsicológica de pessoas com dificuldade de aprendizagem [Dissertação de Mestrado].Brasília: Universidade de Brasília; 2007.

25. Frota S, Pereira LD. Processos temporais em crianças com déficit de consciência fonológica. Rev Iberoam Educac. 2004, 33(9):1-9. 